\title{
Assessment of Hand Hygiene Attitude, Knowledge and Practice among Health Science Students in Aden University
}

\author{
Zeyad A. Sallami \\ Consultant General Surgeon, Bronglais Hospital, Aberystwyth, Wales, UK \\ Email: Alsallami23@hotmail.com
}

How to cite this paper: Sallami, Z.A. (2016) Assessment of Hand Hygiene Attitude, Knowledge and Practice among Health Science Students in Aden University. Journal of Biosciences and Medicines, 4, 25-32.

http://dx.doi.org/10.4236/jbm.2016.49003

Received: August 7, 2016

Accepted: September 13, 2016

Published: September 16, 2016

Copyright $\odot 2016$ by author and Scientific Research Publishing Inc. This work is licensed under the Creative Commons Attribution International License (CC BY 4.0).

http://creativecommons.org/licenses/by/4.0/

\begin{abstract}
Background: A proper hand washing of community health care worker ( $\mathrm{CHCW}$ ) is universally accepted as an important factor in reducing Heath Care Associated Infections (HCAIs). This cross sectional study was done to assess the knowledge, the attitude and practice on hand hygiene among health science students in Aden University. Method: 329 participants from medical, dental and nursing schools completed the questionnaire ( $43.8 \%$ female and $56.2 \%$ males). $1^{\text {st }}, 2^{\text {nd }}$ and internship years were excluded from the study. The questionnaire is adapted from World Health Organization (WHO) tools for hand hygiene surveys. Epi info., an epidemiological software from the Centre of Disease Control (CDC), was used for data analysis. Results: Responses of the participants in the study indicated that the majority of the students were aware of Hand hygiene $(>90 \%)$ and $60 \%$ have had some sort of training. There is an obvious lack of knowledge on hand movement i.e. my five moments of hand hygiene, particularly on the main source and route of cross contamination with pathogens in hospital setting. In hand hygiene practice, $95 \%$ of participants wash their hands before and after food intake, $85 \%$ of participants use soap and water, but $8 \%$ of participants use only water and very few use alcohol base cleansing agent. $84 \%$ of participants wash their hands after handling patient. There was no significant difference between males and females in hand hygiene practice. Conclusion: The study on assessment of knowledge, attitude \& practice of students of medical sciences revealed that almost all participants were aware of hand hygiene. However, at this stage of assessment, knowledge and practice are below $100 \%$ score for the correct response in particular knowledge and practice.
\end{abstract}

\section{Keywords}

Hand Hygiene, Health Care Associated Infection 


\section{Introduction}

Patient protection against Health Care Associated Infections (HCAIs) is becoming urgent and challenging as a patient safety issue. In 2004, World Health Organization (WHO) launched the World alliance on patients' safety, and a number of initiatives were formulated that together formed the elements of patient safety strategy. SAFE LIFE-Clean your Hands is one of these initiatives. Hand cleaning is a simple and inexpensive procedure. Studies show that HCAIs rate can be reduced by $60 \%$ if health care workers (HCWs) comply with proper hand cleaning [1]. Therefore, implementation of standard precautions for all patients all the time is the key to preventing the spread of most microorganisms and Multi-Drug Resistant organisms (MDROs).

In a review of studies done at multinational Centre's Worldwide [1], the most common bacteria causing Health-Care Associated infections (HCAIs) were: (MRSA) Methicillin resistant Staphylococcus aureus, (VREV) vancomycin resistant Enterococci spp. and (ESBL) Extended-spectrum beta ( $\beta$ )-lactamase gram-negative organisms [2]. The emergence of resistance in these microorganisms has mainly been caused by an inappropriate use of antibiotics in general and the use of broad spectrum antibiotics in particular. In addition, the spread of MDROs in health-care settings is common and occurs mostly via HCWs contaminated hands [3].

At any time, over 1.4 million people worldwide are suffering from infections acquired in hospital. In the developed world: 5 - 10 per cent of patients acquire one or more infections [1], and it may reach 44 per cent in developing countries; the risk of health care associated infection is 2 to 20 times higher than in developed countries.

A comprehensive WHO Report on the burden of endemic health care-associated infection worldwide issued on $5^{\text {th }}$ of May 2011 which highlights the importance of HCAIs in different settings and related risk factors describes advantages and challenges of HCAIs [4].

\section{Method \& Materials}

A cross sectional study among Medical, Dental and Nursing students in the University of Aden and Dr. Nasher Higher Health Institute with an estimated total population of 5000 students. Sample of 340 participants as calculated using stat calc. at frequency $50 \%$ and confidence limit of 5\%, were randomly selected using the lists of the students from respective faculties and applying the simple random sample and using random table without replacement, only 329 (144 females (43.8\%) and 185 males (56.2\%) respondents could complete the questionnaire. The questions were selected from WHO model for assessing Knowledge, Attitude and practices for SAVE LIFE Clean YOUR HAND initiative [5] as shown below:

Assessing attitude questions:

1) Hand hygiene practices prevents cross contamination and protect individual from getting infection,

2) Will educate their friends/kith/kin to practice hand hygiene,

3) Feeling experienced whenever a hand hygiene procedure is done. 
Assessing knowledge question:

1) Aware of the hand hygiene practice,

2) Have you received any formal training in hand hygiene in the campus,

3) Main route of cross transmission of potentially harmful germs between patients in a health care facility,

4) Most frequent source of germs responsible for health care associated infections,

5) Actions of hand hygiene that prevents transmission of germs to the patients,

6) Actions of hand hygiene that prevents transmission of germs to the health care worker.

Assessing practice questions:

1) Hand hygiene practices prevents cross contamination and protect individual from getting infection,

2) Will educate their friends/kith/kin to practice hand hygiene,

3) Feeling experienced whenever a hand hygiene procedure is done.

Analysis of data and graphics was done using CDC Epi Info 3.4.5 and Microsoft spread sheet Excel 2013, Approval to conduct the study was obtained from the Faculty of Medicine, the Faculty of Dentistry of Aden University and Dr. Nasher Institute in Aden. The study was conducted during the last semester of the academic year 2014-15. Students who were in their $1^{\text {st }} \& 2^{\text {nd }}$ or internship were excluded from the study. The questionnaire was divided into the three parts. Each part aimed at assessing the knowledge of the participants, the Attitude and the Practice of hand hygiene respectively.

\section{Results}

Out of a total sample of 329 participants $144(43.8 \%)$ were female, they are outnumbered by 41 males, (185 (56.2\%). Responses of the groups were analysed as one cluster and no differentiation between the different professions. In the hand hygiene practice responses were tabulated for each question according to gender category (Figure 1). In all cases, it shows only Yes responses for convenience.

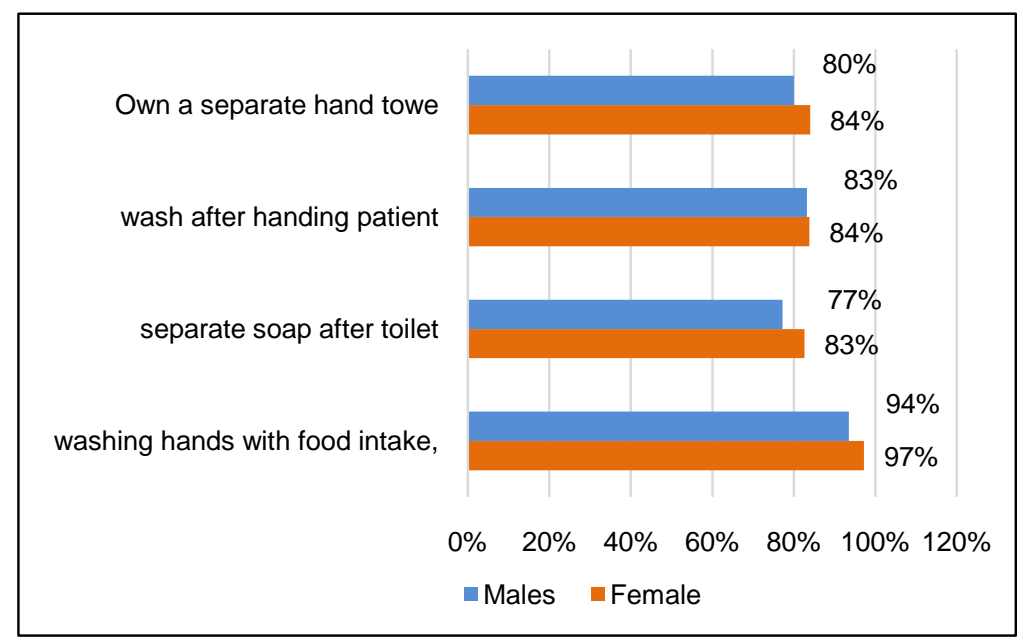

Figure 1. Male and female responses on hand hygiene practice. 


\subsection{Assessing Attitude}

Over $90 \%$ of participant responded YES for the first and the second question (Table 1). The third question was a multiple choice question expressing attitude when hand hygiene procedure is practiced. $3.0 \%$ said they don't care, $42 \%$ they feel safe and secure and $41 \%$ do it as a routine. At extreme $3.0 \%$ do not care and $0.6 \%$ practice hand hygiene only when dirt is obvious in the hands.

\subsection{Knowledge Assessment}

This part includes the five moments, the route of cross contamination and the main route of spreading infection. But first in exploring whether the participants are aware of hand hygiene practice and if they have had any sort of training in hand hygiene practice. Table 2 shows that $98 \%$ are aware of hand hygiene practice, but only $53 \%$ had some sort of training. Responses on the main route of cross contamination of HCAIs among patients, $51 \%$ got the correct answer which implicate the hands of HCWs as source of contamination were not clean.

In evaluating participants' knowledge, the questions tried to focus on the important issues which the health worker should know. In particular, the main sources of contamination and the most frequent route of spreading HCAIs to patients in health care service setting. $51.5 \%$ think that the main route of spreading cross contamination are the hands of HCW when not clean, 26\% think it is the circulating air and followed by $15.5 \%$ think it is exposure to colonized surfaces. Only $6.4 \%$ said that the main route is haring non-invasive instruments. As for the main source, the majority, $51.1 \%$, believe that the main source is the hospital environment, $21 \%$ think it is the germs already present on the patient followed by hospital air, 15\%, and hospital water system, $8.5 \%$.

Table 1. Attitude response assessments.

\begin{tabular}{cc}
\hline & Percentage \\
\hline Yes & \\
Hand hygiene forms an important practice to prevent cross infection & $96.70 \%$ \\
Yes & $95.10 \%$ \\
Will educate friends/family to practice hand hygiene & \\
Yes & $92.40 \%$ \\
Feeling experienced whenever a hand hygiene procedure is done & \\
Don't care of any hand hygiene & $3.00 \%$ \\
Feel safe and secure from getting any infections & $13.10 \%$ \\
Habitual-feel it to be routine & $42.20 \%$ \\
Only when dirt is obvious on the hand & $41.00 \%$ \\
\hline
\end{tabular}


Table 2. Responses for hand hygiene knowledge assessment.

\begin{tabular}{|c|c|}
\hline & Percentage $(\%)$ \\
\hline Aware of hand hygiene practice & 97.8 \\
\hline Have you received any formal training in hand hygiene in the campus & 53.2 \\
\hline \multicolumn{2}{|l|}{ Main route of cross transmission } \\
\hline Air circulation & 26.50 \\
\hline Health care worker hands & 51.50 \\
\hline exposure to colonized surface & 15.50 \\
\hline Sharing non-invasive objects & 6.40 \\
\hline \multicolumn{2}{|l|}{ Most frequent source of germs } \\
\hline Germs already present on/in the patient & 21.10 \\
\hline Hospital air & 18.30 \\
\hline Hospital environment & 51.10 \\
\hline Hospital water system & 9.50 \\
\hline \multicolumn{2}{|l|}{ Actions of hand hygiene that prevents transmission of germs to patients } \\
\hline After exposure to the surroundings of a patient & 11.60 \\
\hline Before touching a patient & 66.50 \\
\hline Immediately after a risk of body fluid exposure & 9.80 \\
\hline Immediately before a clean/aseptic procedure & 12.20 \\
\hline \multicolumn{2}{|l|}{ Action of hand hygiene that prevent transmission of germs to health care worker } \\
\hline After touching a patient & 46.60 \\
\hline After exposure to the surrounding of a patient & 17.10 \\
\hline Immediately before after a clean/aseptic procedure & 26.20 \\
\hline Immediately after a risk of body fluid exposure & 10.10 \\
\hline
\end{tabular}

Questions on identification of the hand action that is intended to prevent cross infection to patient and those actions intended to protect health workers from getting infection i.e. doing hand washing. On hand actions that protect health workers $46.6 \% \mathrm{im}$ mediately after touching the patient, $17 \%$ immediately after exposure to the surrounding and $10 \%$ after the risk of exposure to body fluid. These are the correct answers. On the actions of that is intended for preventing cross infections the respondent chose the correct choice but the percentages of respondents vary. The first question on hand washing habits was washing hand before and after food, (95\%) was positive and less on washing hands after handling each patient (83.5\%), 79\% use soap and water when washing hands after using the toilet and $95 \%$ have a separate hand towel. On the choice of an agent for hand hygiene, the majority use soap and water. 


\subsection{Assessment on Hand Hygiene Practice}

This part (Table 3) deals with practical aspects of daily life. The majority of respondents (95\%) wash their hands before and after food intake, $85 \%$ uses soap and water, but a considerable number (8\%) use only water and very few uses alcohol base cleansing agent. $84 \%$ wash their hands after handling patients. Above $80 \%$ keep their own soap and towels after using the toilet. Figure 1 shows male and female responses on hand hygiene practice, there is marginal difference in washing hands before and after food intake and other practices, both cases have an odd of 0.4 and an odd ratio of 1 showing no significant differences in both sexes.

\section{Discussion}

The objective of this survey was to assess the knowledge, attitude and the practice of the medical, dental and nursing students in the Hand hygiene as related to HCAIs control in a health facility setup. This step is necessary for preparing future health workers on the right path of patients' safety practice. Many medical faculties and health institutions in the East Mediterranean Region (EMRO) will adapt WHO curriculum guidelines to be included in training of medical students. The results of the survey show reasonably good responses towards the importance of hand hygiene. However, this attitude is not reflected in their practice or knowledge about hand hygiene. The attitude of HCWs must be reinforced by evidence based facts about the dangerous consequences of unclean practice, in particular hand hygiene. Early training and practical demonstration of students in their early medical education is absolutely necessary.

Table 3. Hand hygiene practice.

Percentage (\%)

1) Habit of washing hands before and after food intake

YES

$95.10 \%$

2) Choice of agent hand hygiene

Alcohol

$4.00 \%$

Other antimicrobial agents

$3.60 \%$

Soap and water

$84.50 \%$

Water

$7.90 \%$

3) Own a separate soap for washing hands after toilet

YES

$79.60 \%$

4) Habit of hand wash after handing each and every patient

YES

$83.50 \%$

5) Own a separate hand towel

YES

$81.80 \%$ 
The knowledge component of the assessment focuses on three aspects of hand hygiene:

1) The source of the dangerous pathogens which invariably the germs that already exist on the body of the patients and the health worker.

2) The route of cross infection to patients and the health worker which is always the unclean hand of HCWs.

3) The action of hands during patient care procedures. Emphasizing "My five moments for hand hygiene defines the key moments when health-care workers should perform hand hygiene.

This evidence-based, field-tested, user-centred approach is designed to be easy to learn, logical and applicable in a wide range of settings. This approach recommends health-care workers to clean their hands:

1) Before touching a patient,

2) Before clean/aseptic procedures,

3) After body fluid exposure/risk,

4) After touching a patient, and

5) After touching patient's surroundings.

The results in various responses reflect uncertainty in all three aspects of knowledge. This again emphasises the need for further training. The results for practice shows typical results of incompliance with hand washing before and after food or after a toilet visit and perhaps the unavailability of cleaning materials which often determine the choice of the disinfecting agent. This needs separate survey by itself.

By their very nature, infections are caused by many different factors related to systems and processes of care provision as well as to human behaviour that is conditioned by education, political and economic constraints on systems and countries, and often on societal norms and beliefs. Hand hygiene, a simple action, perhaps, but the lack of compliance among health-care providers and in some occasions unawareness is problematic worldwide. On the basis of research into the aspects influencing hand hygiene compliance and best promotional strategies.

WHO Alliance for patients' safety surveys demonstrated that noncompliance due to lack of hand hygiene utilities at hospital level. Encouraging hospitals and health-care facilities to adopt these Guidelines, including the "My 5 Moments for Hand Hygiene" approach, will contribute to a greater awareness and understanding of the importance of hand hygiene. While system change is a requirement in most places, sustained change in human behaviour is even more important and relies on essential peer and political support. It is therefore control of HCAIs and consequently hand hygiene is not an option, it is human right. Training and practical demonstration for health workers early in their career is important before they get used to the wrong way and becomes a habit.

\section{Conclusion}

The study on assessment of knowledge, attitude \& practice of students of medical sciences revealed that almost all participants were aware of hand hygiene, and this was an 
indication that any future training would reinforce safe practice of hand hygiene as an important component of controlling HCAIs. However, at this stage of assessment, knowledge and practice are below $100 \%$ score for the correct response in particular for main source of infection, the main route of spreading the infection and the five moments for hand hygiene.

\section{References}

[1] World Health Organization. WHO Guidelines on Hand Hygiene in Health Care. http://www.who.int/gpsc/information_centre/hand-hygiene-2009/en/

[2] EMDRO Literature Review. Evidence of Hand Hygiene to Reduce Transmission and Infections by Multidrug Resistant Organisms in Health-Care Settings. http://www.who.int/gpsc/5may/MDRO_literature-review.pdf

[3] Chemaly, R.F., Simmons, S., Dale Jr., C., Ghantoji, S.S., Rodriguez, M., Gubb, J., Stachowiak, J. and Stibich, M. (2014) The Role of the Healthcare Environment in the Spread of Multidrug-Resistant Organisms: Update on Current Best Practices for Containment. Therapeutic Advances in Infectious Disease, 2, 79-90.

[4] (2011) Report on the Burden of Endemic Health Care-Associated Infection Worldwide. World Health Organization, Geneva. http://apps.who.int/iris/bitstream/10665/80135/1/9789241501507_eng.pdf

[5] WHO. Tools for Evaluation and Feedback. http://www.who.int/gpsc/5may/tools/evaluation_feedback/en/

Submit or recommend next manuscript to SCIRP and we will provide best service for you:

Accepting pre-submission inquiries through Email, Facebook, LinkedIn, Twitter, etc.

A wide selection of journals (inclusive of 9 subjects, more than 200 journals)

Providing 24-hour high-quality service

User-friendly online submission system

Fair and swift peer-review system

Efficient typesetting and proofreading procedure

Display of the result of downloads and visits, as well as the number of cited articles

Maximum dissemination of your research work

Submit your manuscript at: http://papersubmission.scirp.org/ 